Bulletin of the Section of Logic

Volume 45/1 (2016), pp. 33-51

http://dx.doi.org/10.18778/0138-0680.45.1.03

Mirjana Ilic ${ }^{1}$

\title{
AN ALTERNATIVE NATURAL DEDUCTION FOR THE INTUITIONISTIC PROPOSITIONAL LOGIC
}

\begin{abstract}
A natural deduction system $\mathcal{N} \mathcal{I}$, for the full propositional intuitionistic logic, is proposed. The operational rules of $\mathcal{N} \mathcal{I}$ are obtained by the translation from Gentzen's calculus $L J$ and the normalization is proved, via translations from sequent calculus derivations to natural deduction derivations and back.

Keywords: natural deduction; intuitionistic logic

\section{Introduction}

It is well-known that sequent formulations of some substructural logics can be obtained from Gentzen's calculus $L J$, by rejecting or restricting some of the structural rules, whereas operational rules need not differ from the operational rules that are given for intuitionistic logic in $L J$ (see [1]). On the other hand, Gentzen's natural deduction calculus $N J$ [3] doesn't have the similar property. Our motive in formulating natural deduction system $\mathcal{N} \mathcal{I}$ is to set up an intuitionistic natural deduction system, from which natural deduction systems of some substructural logics can be obtained, by getting the control over the discharge of assumptions, whereas operational rules need not differ from the operational rules in $\mathcal{N} \mathcal{I}$ (as for sequent calculi).
\end{abstract}

\footnotetext{
${ }^{1}$ This work is supported by the Ministary of Science and Technology of Serbia, grant number ON174026.
} 
It is well-known that the natural deduction rule $\wedge-I$ :

$$
\frac{\alpha \beta}{\alpha \wedge \beta}
$$

can be of one of the following forms:

$\begin{array}{ccccc}\Gamma & \Gamma & \Gamma & \Delta \\ \vdots & \vdots & & \vdots & \vdots \\ \Gamma & \frac{\alpha}{\alpha \wedge \beta} & \text { or: } & \frac{\alpha}{\alpha \wedge \beta}\end{array}$

where $\alpha$ denotes a natural deduction derivation of $\alpha$ with open assumptions from $\Gamma$. Open assumptions are called the contexts of the rules, so the rule on the left is usually referred to as a context-sharing rule and the rule on the right, as a context-independent rule ( $\Gamma$ and $\Delta$ which appear in the later, may be different). We have the similar notion for the rule $\rightarrow-E$ :

$$
\frac{\alpha \quad \alpha \rightarrow \beta}{\beta} .
$$

For the plain intuitionistic logic, contexts of those rules are irrelevant, therefore, in intuitionistic natural deduction calculi, operational rules are either context-sharing, as e.g. in NJ [8], or context-independent, as e.g. in Gentzen's NJ and von Plato's natural deduction [6]. On the other hand, this is not the same in Gentzen's sequent calculus $L J$, where two types of operational rules are present: the rule which corresponds to $\wedge-I$, i.e. the rule $\wedge-I S$, is context-sharing and the rule $\rightarrow-I A$, which corresponds to $\rightarrow-E$, is context-independent:

$$
\wedge-I S: \frac{\Gamma \vdash \alpha \quad \Gamma \vdash \beta}{\Gamma \vdash \alpha \wedge \beta} \rightarrow-I A: \frac{\Gamma \vdash \alpha \quad \beta, \Delta \vdash \Lambda}{\alpha \rightarrow \beta, \Gamma, \Delta \vdash \Lambda}
$$

( $\Lambda$ is either singleton or empty). 
In this paper we give a natural deduction system $\mathcal{N} \mathcal{I}$, with both types of operational rules, context-sharing and context-independent ones. We obtain them by a translation of Gentzen's operational $L J$-rules, into natural deduction. The idea of formulating natural deduction systems for intuitionistic logic, by translating sequent calculi, is not new (see e.g. [5], [6]). Actually, the technique employed here is substantially that given by Negri and Plato in [5], [6]. However, sequent calculi in [5], [6] are with context-independent rules only, unlike $L J$, therefore our natural deduction system differs from those in [5], [6].

Moreover, instead of usual assumptions, i.e. initial formulae $\alpha$, we use general assumptions, in the form of initial rules:

$$
\frac{\alpha, \alpha_{1}, \ldots, \alpha_{n}}{\alpha}
$$

in $\mathcal{N} \mathcal{I}$. As a consequence, $\mathcal{N} \mathcal{I}$-derivations are free from vacuously discharged assumptions, meaning that every formula which is discharged by the application of some inference rule, really occurs in the upper part of at least one initial rule in that derivation. Below, we give two derivations of the formula $\alpha \rightarrow(\beta \rightarrow \alpha)$. In the derivation on the left, we use initial formulae, whereas in the derivation on the right, we use initial rules:

(1) (2)

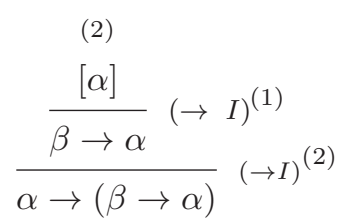

$$
\frac{\frac{[\beta],[\alpha]}{\alpha}}{\frac{\beta \rightarrow \alpha}{\alpha \rightarrow(\beta \rightarrow \alpha)}(\rightarrow I)^{(1)}}\left({ }^{(2)}\right.
$$

We note that, in the derivation on the left, the formula $\beta$ is vacuously discharged (we also say cancelled), by the application of the rule $(\rightarrow I)$, with the discharge label (1), unlike in the derivation on the right, where the assumption $\beta$ really occurs. Moreover, the initial formula $\alpha$ in the derivation on the left, stands in for the derivation of $\alpha$ from $\alpha$, i.e. for the derivation $\frac{\alpha}{\alpha}$, therefore it acts as both, an assumption and a conclusion. On the other hand, in the derivation on the right, every formula acts either as an assumption or as a conclusion. 
It should be mentioned that the use of generalized assumptions, in formulating natural deduction for intuitionistic propositional logic, is not new. E.g., in the sequent-style natural deduction NJ, in [8], axioms are of the form:

$$
\Gamma, \varphi \vdash \varphi .
$$

However, our system $\mathcal{N} \mathcal{I}$ and NJ are different systems, as mentioned above.

The paper is organized as follows. In Section 2, we give the sequent calculus formulation $G I$ of the full propositional intuitionistic logic, with the property that the structural rules of cut and weakening are admissible in it. In Section 3, we present the natural deduction system $\mathcal{N} \mathcal{I}$. In Section 4 , we prove that $\mathcal{N} \mathcal{I}$ is sound and complete for the full propositional intuitionistic logic. In the closing section we give some remarks.

\section{Sequent calculus $G I$}

Let $\mathcal{L}$ be a language of the propositional calculus with the propositional constant $\perp$ and the binary connectives $\rightarrow, \wedge$ and $\vee$. Formulae of $\mathcal{L}$ are defined inductively, as usual. We shall use $\alpha, \beta, \gamma, \varphi, \ldots$ as schematic letters for formulae of $\mathcal{L}$. As usual, $\neg \alpha$ can be defined as $\alpha \rightarrow \perp$. Greek capitals $\Gamma, \Pi, \Theta, \ldots$ will denote finite (possibly empty) multisets (lists without order) of formulae.

Sequents are expressions of the form $\Gamma \vdash \gamma$.

$G I$ has the following postulates: Axioms:

$$
\Gamma, \alpha \vdash \alpha \quad \perp, \Gamma \vdash \gamma
$$

Structural rules:

contraction:

$$
\frac{\Gamma, \alpha, \alpha \vdash \gamma}{\Gamma, \alpha \vdash \gamma}(\mathrm{W}) \quad \frac{\Gamma \vdash \gamma}{\Gamma, \alpha \vdash \gamma}
$$

cut:

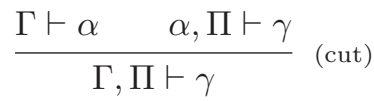

Operational rules:

$$
\frac{\Gamma \vdash \alpha \quad \Pi, \beta \vdash \gamma}{\Gamma, \Pi, \alpha \rightarrow \beta \vdash \gamma}(\rightarrow 1) \quad \frac{\alpha, \Gamma \vdash \beta}{\Gamma \vdash \alpha \rightarrow \beta}(\rightarrow \mathrm{r})
$$


An Alternative Natural Deduction for the Intuitionistic Propositional Logic 37

$$
\begin{aligned}
& \frac{\Gamma, \alpha \vdash \gamma}{\Gamma, \alpha \wedge \beta \vdash \gamma} \quad \frac{\Gamma, \beta \vdash \gamma}{\Gamma, \alpha \wedge \beta \vdash \gamma}\left(\wedge \text { l) } \quad \frac{\Gamma \vdash \alpha}{\Gamma \vdash \alpha \wedge \beta}(\wedge \text { r })\right. \\
& \frac{\Gamma, \alpha \vdash \gamma \quad \Gamma, \beta \vdash \gamma}{\Gamma, \alpha \vee \beta \vdash \gamma}(\vee \mathrm{l}) \quad \frac{\Gamma \vdash \alpha}{\Gamma \vdash \alpha \vee \beta} \frac{\Gamma \vdash \beta}{\Gamma \vdash \alpha \vee \beta}(\vee \mathrm{r})
\end{aligned}
$$

There are some minor differences between $G I$ and some other sequent calculi for intuitionistic propositional logic, e.g. $L J$ [3], G2i [9] and $G 0 i$ [6]. However, all of them are mutually equivalent.

The admissibility of the rule of cut in GI, can be proved by a standard cut-elimination procedure, via the elimination of the rule of multicut. I.e., instead of cut we eliminate the multiple cut rule of the following form:

$$
\frac{\Gamma \vdash \alpha \quad \alpha, \ldots, \alpha, \Pi \vdash \gamma}{\Gamma, \ldots, \Gamma, \Pi \vdash \gamma} \text { (mcut) }
$$

Moreover, the rule of weakening is also an admissible rule in $G I$ :

LEMMA 1. Every cut-free GI-derivation can be transformed into a cutfree GI-derivation of the same endsequent, in which no weakening occurs.

Proof: It is easy to show that weakening never needs to follow immediately any other rule in a cut-free $G I$-derivation.

Definition. Cut-free $G I$-derivations with no applications of the rule of weakening, will be called normal GI-derivations.

Corollary 2. Every GI-provable sequent has a normal GI-derivation.

\section{Natural deduction $\mathcal{N} \mathcal{I}$}

We formulate the natural deduction system $\mathcal{N} \mathcal{I}$, by a translation of axioms and logical rules of $G I$ into inference rules of $\mathcal{N} \mathcal{I}$.

First we consider the axioms of $G I$. The meaning of $\alpha, \Gamma \vdash \alpha$, i.e. of $\alpha, \alpha_{1}, \ldots, \alpha_{n} \vdash \alpha$ (the multiset $\Gamma=\alpha_{1}, \ldots, \alpha_{n}$ is possibly empty) is that from $\alpha, \alpha_{1}, \ldots, \alpha_{n}$ we can derive $\alpha$. In the natural deduction notation, this is written as:

$$
\frac{\alpha, \alpha_{1}, \ldots, \alpha_{n}}{\alpha},
$$

which is one of our initial rules of $\mathcal{N} \mathcal{I}$. 
The other axiom in $G I$ is $\perp, \alpha_{1}, \ldots, \alpha_{n} \vdash \gamma$, which gives another initial rule of $\mathcal{N} \mathcal{I}$ :

$$
\frac{\perp, \alpha_{1}, \ldots, \alpha_{n}}{\gamma} .
$$

The meaning of the rule $(\rightarrow 1)$ in $G I$ is that if there is a derivation of $\alpha$ from $\Gamma$ and there is a derivation of $\gamma$ from $\Pi, \beta$, then there is a derivation of $\gamma$ from $\Gamma, \Pi, \alpha \rightarrow \beta$. In the natural deduction notation, this is written as:

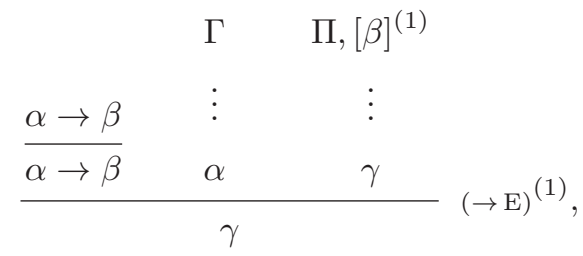

which is the elimination of implication rule, denoted by $(\rightarrow \mathrm{E})$ in $\mathcal{N} \mathcal{I}$. With $[\beta]$ we denote, as usual, the discharging of the assumption $\beta$. To indicate what is discharged and where, we write the little number within parentheses, here (1), next to the discharged assumption $\beta$ and next to the mnemonic symbol for the rule applied, $(\rightarrow \mathrm{E})$. Those numbers will be called discharge labels and they must be fresh in the derivation so far constructed. We note that this rule is almost the same as von Plato's rule $(\supset \mathrm{E})$ in $[6]$. The only difference is due to our use of initial rules instead of initial formulae, i.e. that instead of von Plato's initial formula $\alpha \rightarrow \beta$ we use the initial rule $\frac{\alpha \rightarrow \beta}{\alpha \rightarrow \beta}$. This is the general formulation of the implication elimination rule, and it differs from the corresponding Gentzen's rule, which is the special elimination rule. All other elimination rules of $\mathcal{N} \mathcal{I}$ will also be given in their general form.

The rule $(\rightarrow 1)$ is a two-premises context-independent rule. On the other hand, the rules $(\wedge \mathrm{r})$ and $(\vee \mathrm{l})$ are two-premises context-sharing rules. To obtain the corresponding natural deduction rules, we need to assign labels to assumptions, as in [4]. We shall call them the assumption labels. Formulae with the same assumption label are treated as if they were the same formulae. This means that when a formula with a label is discharged, all formulae with the same label are discharged at the same time. We use integers for assumption labels (to distinguish them from discharge labels, 
we write them without parentheses). The natural deduction formulation of the rule $(\wedge \mathrm{r})$ is:

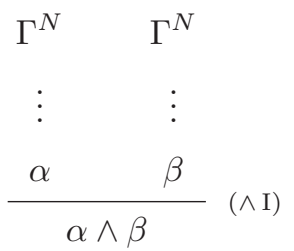

By the application of this rule, the corresponding formulae from $\Gamma$ in the derivation of $\alpha$, and from $\Gamma$ in the derivation of $\beta$, are assigned with the same assumption labels. This is indicated by ${ }^{N}$ in $\Gamma^{N}$ (if a formula from $\Gamma$ in the derivation of $\beta$, already has a label, assigned to it earlier in the derivation, then, by the application of this rule, this formula gets a new label, in order to equalize it with the label of its corresponding formula from $\Gamma$ in the derivation of $\alpha$ ).

It should be noted that the use of assumption labels is not new in formulating natural deduction systems. In fact, the use of labels is quite standard in natural deduction systems of substructural logics (see e.g. [2] where labelled natural deduction system for the implicational fragment of the relevant logic $R$ is presented, and [4], for the natural deduction system for intuitionistic linear logic).

Natural deduction $\mathcal{N} \mathcal{I}$

Initial rules:

$$
\frac{\alpha, \alpha_{1}, \ldots, \alpha_{n}}{\alpha}, \quad \frac{\perp, \alpha_{1}, \ldots, \alpha_{n}}{\gamma},
$$

where the multiset $\alpha_{1}, \ldots, \alpha_{n}$ is possibly empty. Formulae from the premises of the initial rules will be called the assumptions.

Logical rules:

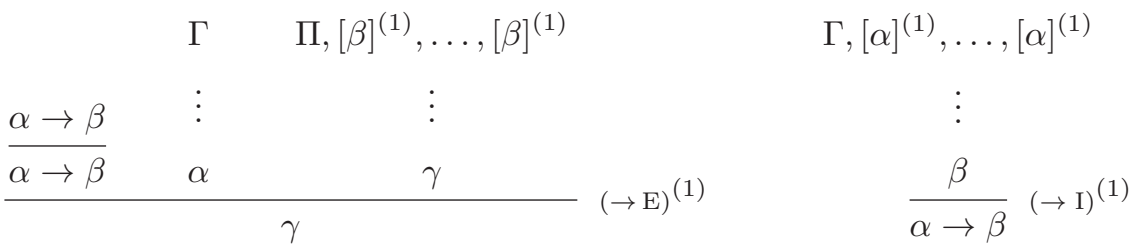



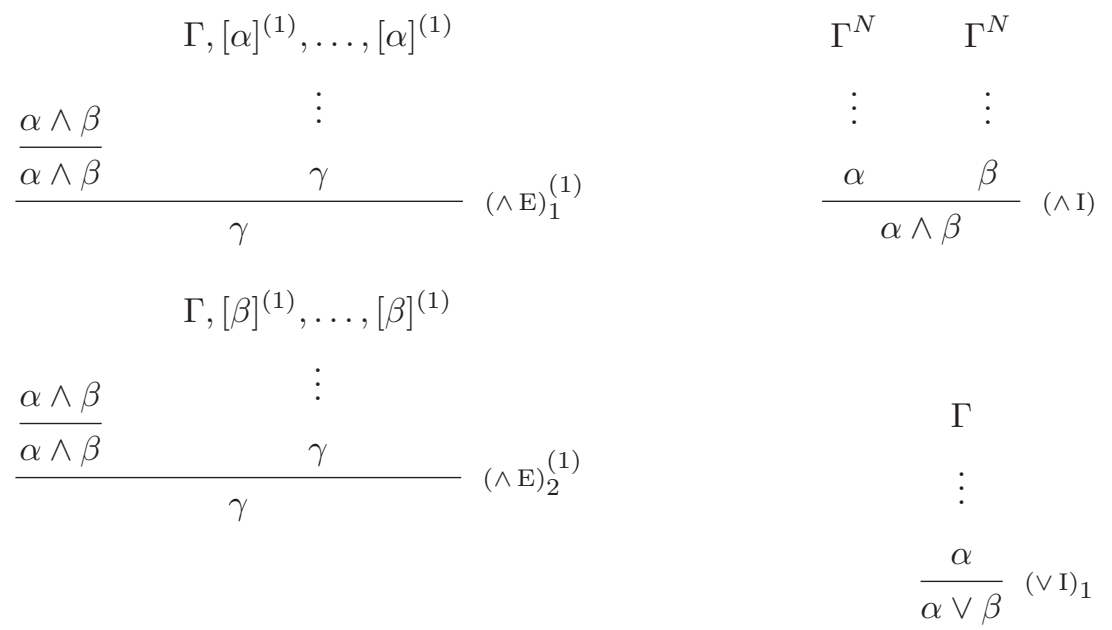

$$
\Gamma^{N},[\alpha]^{(1)}, \ldots,[\alpha]^{(1)} \quad \Gamma^{N},[\beta]^{(2)}, \ldots,[\beta]^{(2)}
$$

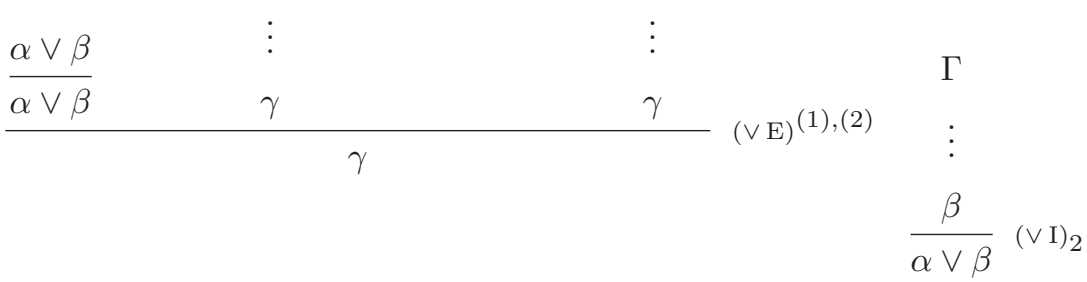

We note that there is no need to formulate the natural deduction rules which correspond to the structural rules of cut and weakening, since it is sufficient to consider normal GI-derivations. The remaining structural rule, the rule of contraction, is implicit in natural deduction, in the form of multiple discharge of assumptions. 
We give an example proof of a distribution law in $\mathcal{N} \mathcal{I}$ :

$$
\begin{aligned}
& 11 \\
& \begin{array}{llllllll}
1 & 2 & 1 & 2 & \not 3 & 4 & \not 3 & 4
\end{array} \\
& \underline{[\alpha]^{(3)},[\beta]^{(1)}} \underline{[\alpha]^{(3)},[\beta]^{(1)}} \underline{[\alpha]^{(3)},[\gamma]^{(2)}} \underline{[\alpha]^{(3)},[\gamma]^{(2)}}
\end{aligned}
$$

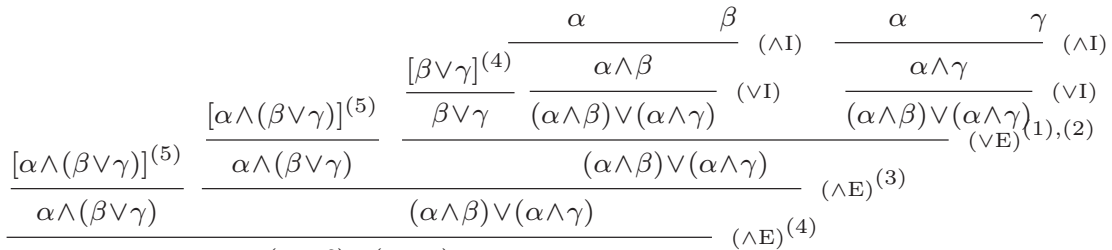

$$
\begin{aligned}
& \frac{\frac{\alpha \wedge(\beta \vee \gamma)}{(\alpha \wedge \beta) \vee(\alpha \wedge \gamma)}}{(\alpha \wedge(\beta \vee \gamma)) \rightarrow .(\alpha \wedge \beta) \vee(\alpha \wedge \gamma)}(\rightarrow \mathrm{I})^{(5)}{ }^{(\wedge \mathrm{E})^{(4)}}
\end{aligned}
$$

The use of general, instead of special elimination rules in natural deduction, allows us to define normal derivations as in [4], [6]:

Definition. A derivation is in normal form when all major premises of elimination rules are conclusions of initial rules.

We note that every $\mathcal{N} \mathcal{I}$-derivation is in normal form.

\section{Equivalence of $G I$ and $\mathcal{N I}$}

$G I$ and $\mathcal{N} \mathcal{I}$ are equivalent, and with this we mean:

Theorem 3. For any formula $\gamma$, there is a derivation of $\vdash \gamma$ in GI iff there is a derivation of $\gamma$ with all assumptions discharged in $\mathcal{N} \mathcal{I}$.

PROOF: We define a translation from a normal $G I$-derivation $\pi$, of a sequent $\vdash \gamma$, to natural deduction, inductively according to the last rule applied.

The last applied rule in $\pi$ can be one of: $(\rightarrow \mathrm{r}),(\wedge \mathrm{r})$ or $(\vee \mathrm{r})$. Then the translation is given as follows:
$\pi_{1}$
$\pi_{1}$
1.1. $\frac{\alpha \vdash \beta}{\vdash \alpha \rightarrow \beta}(\rightarrow \mathrm{r}) \mapsto \frac{[\alpha]^{(1)} \vdash \beta}{\alpha \rightarrow \beta}(\rightarrow \mathrm{I})^{(1)}$

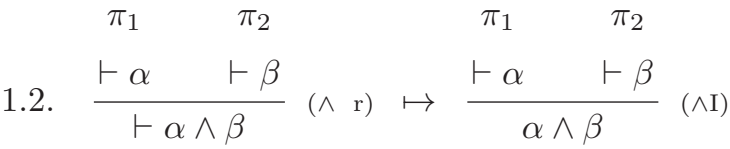


$\begin{array}{cccc}\pi_{1} & \pi_{1} & \pi_{1} & \pi_{1} \\ \text { 1.3. } & \frac{\vdash \alpha}{\vdash \alpha \vee \beta}(\vee \mathrm{r}) \mapsto \frac{\vdash \alpha}{\alpha \vee \beta}(\vee \mathrm{I}) & \frac{\vdash \beta}{\vdash \alpha \vee \beta}(\vee \mathrm{r}) \mapsto \frac{\vdash \beta}{\alpha \vee \beta}(\vee \mathrm{I})\end{array}$

In each case of translation, we write sequent calculus derivation(s) in the upper part, the rule in natural deduction notation and the conclusion of that rule in the lower part. The sequent calculus derivation(s) in the upper part will be called the sequent calculus segments of the translation, noting they are the same as the corresponding sequent calculus derivations given on the left-hand side of $\mapsto$. Those derivations will be translated in the further steps of the translation.

The formula $\alpha$ from $[\alpha]^{(1)} \vdash \beta$ in 1.1, will be an assumption, discharged by the application of the rule $(\rightarrow \mathrm{I})$, in a natural deduction derivation, obtained by the translation. This is the reason why instead of $\alpha$ we write $[\alpha]^{(1)}$ in 1.1., i.e. why we add brackets and the discharge label (1). In the following translations, we shall use $[\Gamma]$ to denote a multiset of bracketed formulae, assigned with discharge labels.

Let $\operatorname{Tr}$ be a translation so far constructed and let one of its sequent calculus segments be the segment $S$, which is given on the left-hand side of $\mapsto$ below. If the last rule in $S$ is an operational rule, then we have:

$\pi_{1}$

$\pi_{1}$

2.1. $\frac{\alpha, \Gamma \vdash \beta}{[\Gamma] \vdash \alpha \rightarrow \beta}(\rightarrow \mathrm{r}) \mapsto \frac{[\alpha]^{(i)},[\Gamma] \vdash \beta}{\alpha \rightarrow \beta}(\rightarrow \mathrm{I})^{(i)}$

provided $(i)$ is new discharge label in the translation so far constructed.

$\pi_{1} \quad \pi_{2}$

2.2. $\frac{\Gamma \vdash \alpha \quad \Pi, \beta \vdash \gamma}{[\Gamma],[\Pi],[\alpha \rightarrow \beta]^{(k)} \vdash \gamma}(\rightarrow 1) \mapsto$

$$
\begin{aligned}
& {[\alpha \rightarrow \beta]^{(k)} \quad \pi_{1} \quad \pi_{2}} \\
& \mapsto \frac{\overline{\alpha \rightarrow \beta} \quad[\Gamma] \vdash \alpha \quad[\Pi],[\beta]^{(i)} \vdash \gamma}{\gamma}(\rightarrow \mathrm{E})^{(i)}
\end{aligned}
$$

provided $(i)$ is new discharge label in the translation so far constructed. 
An Alternative Natural Deduction for the Intuitionistic Propositional Logic 43

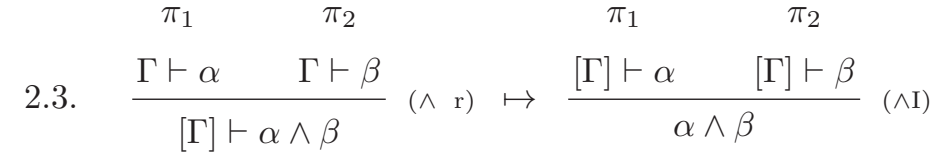

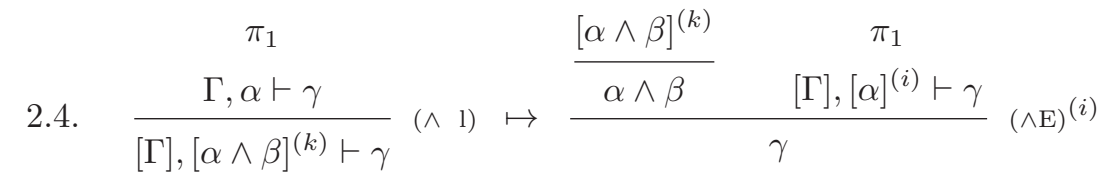

provided $(i)$ is new discharge label in the translation so far constructed. We proceed similarly when the endsequent of $\pi_{1}$ is $\Gamma, \beta \vdash \gamma$.

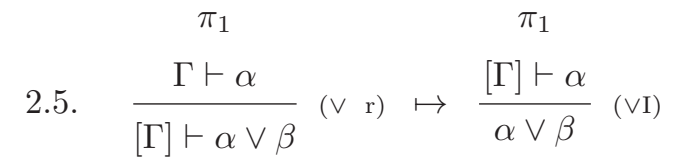

We proceed similarly when the endsequent of $\pi_{1}$ is $\Gamma \vdash \beta$.

$\pi_{1}$ $\pi_{2}$

2.6 .

$$
\begin{aligned}
& \frac{\Gamma, \alpha \vdash \gamma \quad \Gamma, \beta \vdash \gamma}{[\Gamma],[\alpha \vee \beta]^{(k)} \vdash \gamma}(\vee 1) \mapsto
\end{aligned}
$$

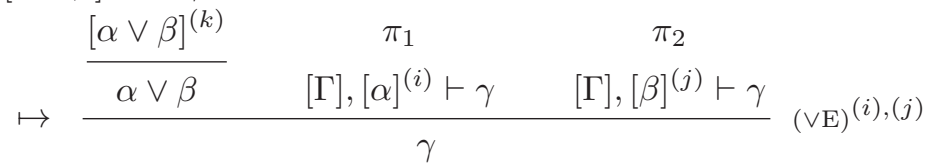

provided $(i)$ and $(j)$ are distinct and new in the translation so far constructed.

If the last rule in $S$ is the structural rule of contraction, then we have:

$\pi_{1}$

2.7. $\frac{\Gamma, \alpha, \alpha \vdash \gamma}{[\Gamma],[\alpha]^{(k)} \vdash \gamma}$ (WE) $\mapsto \quad[\Gamma],[\alpha]^{(k)},[\alpha]^{(k)} \vdash \gamma$

If $S$ is an axiom, then we have:

3.1. $[\alpha]^{(k)},\left[\alpha_{1}\right]^{\left(k_{1}\right)}, \ldots,\left[\alpha_{n}\right]^{\left(k_{n}\right)} \vdash \alpha \mapsto \frac{[\alpha]^{(k)},\left[\alpha_{1}\right]^{\left(k_{1}\right)}, \ldots,\left[\alpha_{n}\right]^{\left(k_{n}\right)}}{\alpha}$

3.2. $[\perp]^{(k)},\left[\alpha_{1}\right]^{\left(k_{1}\right)}, \ldots,\left[\alpha_{n}\right]^{\left(k_{n}\right)} \vdash \gamma \mapsto \frac{[\perp]^{(k)},\left[\alpha_{1}\right]^{\left(k_{1}\right)}, \ldots,\left[\alpha_{n}\right]^{\left(k_{n}\right)}}{\gamma}$. 
Our translation produces natural deduction derivations with discharges fully formalized. On the other hand, the assumption labels which should be assigned by the application of the rules $(\wedge I)$ and $(\vee E)$ are missing. However, they are not needed. Really, the purpose of assumption labels in natural deduction derivations is to identify different occurrences of the same formulae, in order to discharge all of them at the same time, but this is guaranteed by our translation.

Thus, we have proved:

If $\pi$ is a normal GI-derivation of a sequent $\vdash \gamma$, then its translation is an $\mathcal{N} \mathcal{I}$-derivation of $\gamma$ with all assumption discharged (and it is normal, by our definition).

We now define the translation of an $\mathcal{N} \mathcal{I}$-derivation of a formula $\gamma$, with all assumptions discharged, to a $G I$-derivation, inductively, according to the last rule used. Multisets of open assumptions $\Pi, \Gamma$ and $\Theta$, which appear in natural deduction derivations, are possibly empty.

If the last rule of the natural deduction derivation is $(\rightarrow I)$, then we have the following cases. If exactly one occurrence of $\alpha$ is discharged by the application of the rule $(\rightarrow \mathrm{I})$, then:

$$
\begin{aligned}
& \Pi,[\alpha]^{(i)} \quad \Pi, \alpha \\
& \vdots \quad \vdots \\
& \frac{\beta}{\alpha \rightarrow \beta}(\rightarrow \mathrm{I})^{(i)} \mapsto \frac{\beta}{\Pi \vdash \alpha \rightarrow \beta}(\rightarrow \mathrm{r})
\end{aligned}
$$

(Note that if $(\rightarrow \mathrm{I})$ is the last rule of our derivation, then $\Pi$ is empty.)

On the right-hand side of $\mapsto$ we remove the discharge label $(i)$ and brackets in $[\alpha]^{(i)}$, to denote that $\alpha$ is the open assumption in the derivation of $\beta$ from $\Pi, \alpha$.

If at least two occurrences of $\alpha$ are discharged by the application of the rule $(\rightarrow \mathrm{I})$, then: 
An Alternative Natural Deduction for the Intuitionistic Propositional Logic 45

$$
\begin{array}{cc}
\Pi,[\alpha]^{(i)}, \ldots,[\alpha]^{(i)} & \Pi, \alpha, \ldots, \alpha \\
\vdots & \vdots \\
\frac{\beta}{\alpha \rightarrow \beta}\left(^{(\rightarrow \mathrm{I})}{ }^{(i)} \mapsto\right. & \frac{\beta}{\frac{\Pi, \alpha \vdash \beta}{\Pi \vdash \alpha \rightarrow \beta}}(\mathrm{W}),
\end{array}
$$

where double line next to (W) denotes possibly several contractions (at least one).

If the last rule is $(\rightarrow \mathrm{E})$, then we have the following cases:

$$
\begin{aligned}
& \Theta \quad \Pi,[\beta]^{(i)} \quad \Theta \quad \Pi, \beta
\end{aligned}
$$

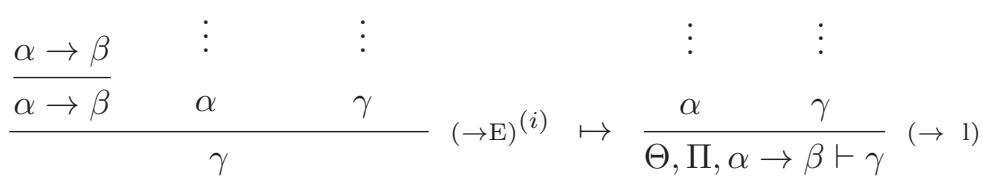

$$
\begin{aligned}
& \Pi, \beta, \ldots \beta \\
& \Theta \quad \Pi,[\beta]^{(i)}, \ldots,[\beta]^{(i)} \\
& \Theta \quad \vdots
\end{aligned}
$$

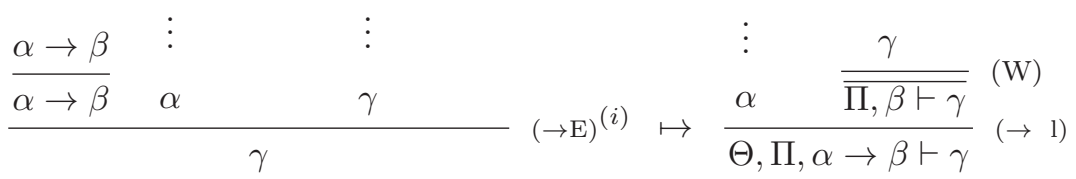

If the last rule is $(\wedge \mathrm{I})$, then we have:

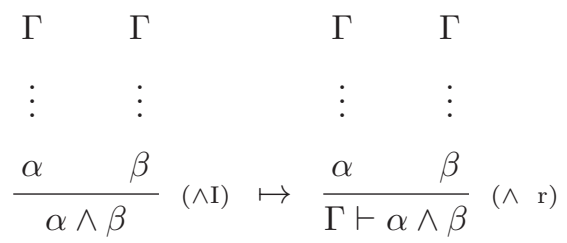

(Note that we omit assumption labels.) If the last rule is $(\wedge \mathrm{E})$, then we have the following cases: 


$$
\begin{aligned}
& \Gamma,[\alpha]^{(i)} \quad \Gamma, \alpha
\end{aligned}
$$

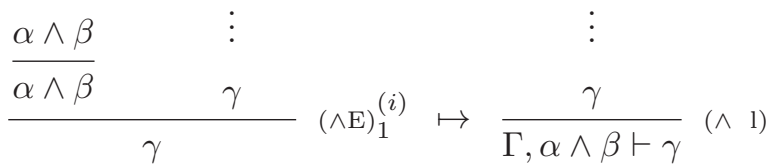

$$
\begin{aligned}
& \Gamma,[\alpha]^{(i)}, \ldots,[\alpha]^{(i)} \quad \Gamma, \alpha, \ldots, \alpha
\end{aligned}
$$

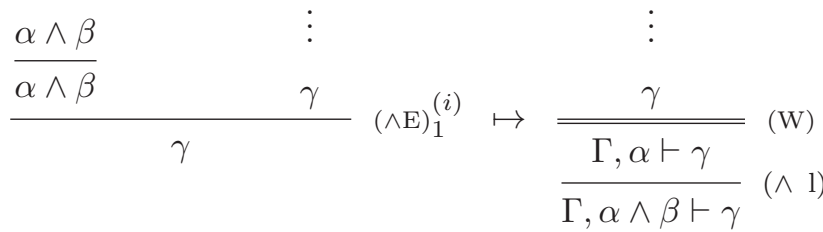

We can proceed similarly for the rule $(\wedge \mathrm{E})_{2}$.

If the last rule is $(\vee \mathrm{I})$, then we have:

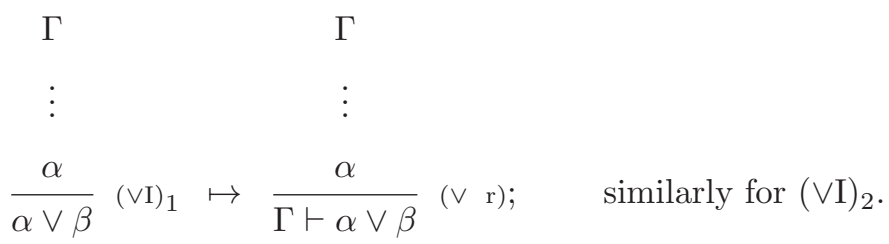

If the last rule is $(\mathrm{VE})$, then we have the following cases:

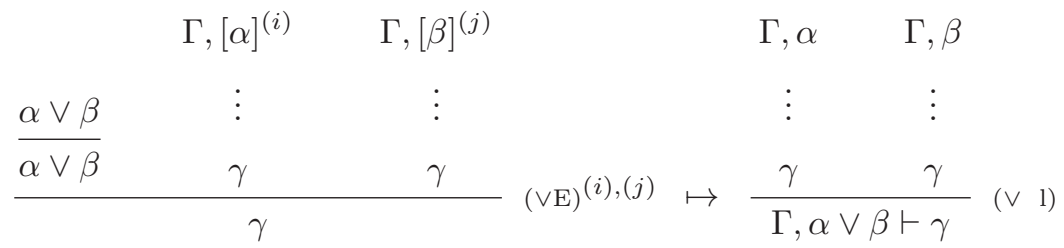




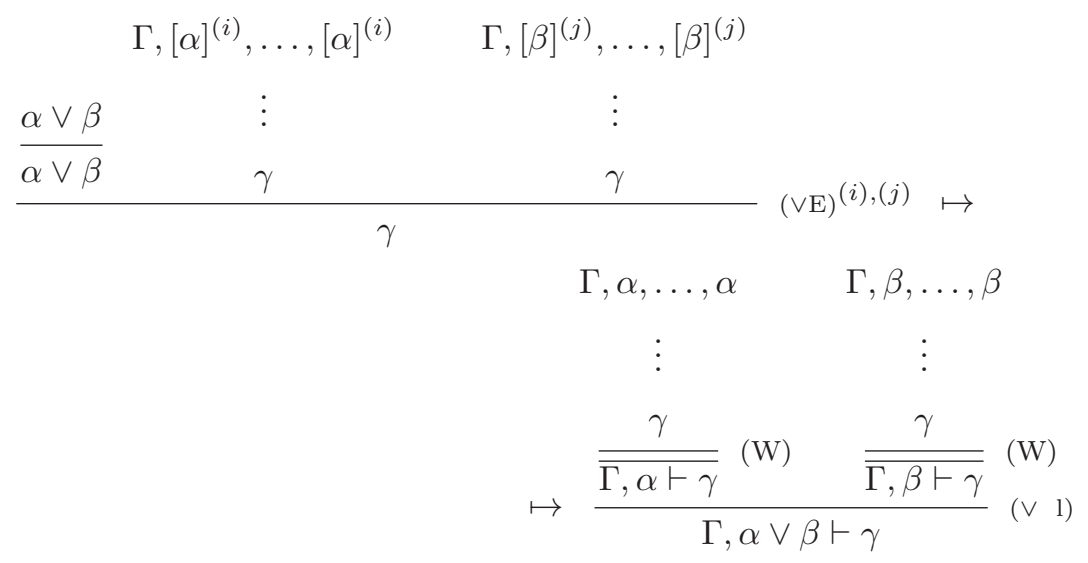

If the last rule is an initial rule, then we have:

$$
\begin{aligned}
& \frac{\alpha, \alpha_{1}, \ldots, \alpha_{n}}{\alpha} \mapsto \alpha, \alpha_{1}, \ldots, \alpha_{n} \vdash \alpha \\
& \frac{\perp, \alpha_{1}, \ldots, \alpha_{n}}{\gamma} \mapsto \quad \perp, \alpha_{1}, \ldots, \alpha_{n} \vdash \gamma
\end{aligned}
$$

We note that the sequent calculus derivation obtained by this translation is normal, thus we have proved:

If $d$ is an $\mathcal{N} \mathcal{I}$-derivation of $\gamma$ with all assumptions discharged, then its translation is a normal GI-derivation of $\vdash \gamma$.

Having the translations from normal $G I$ derivations to $\mathcal{N} \mathcal{I}$-derivations and vice versa, we can now complete the proof of our Theorem. Let $\pi$ be a $G I$-derivation of $\vdash \gamma$. Then there is a normal $G I$-derivation $\pi^{n}$ of $\gamma$. By the translation defined, the translation of $\pi^{n}$ is an $\mathcal{N} \mathcal{I}$-derivation of $\gamma$, with all assumptions discharged. Conversely, let $d$ be an $\mathcal{N} \mathcal{I}$-derivation of $\gamma$, with all assumptions discharged. Then its translation is a (normal) $G I$-derivation.

COROllary 4. In an $\mathcal{N} \mathcal{I}$-derivation of $\gamma$ with all assumptions discharged, each formula in the derivation is a subformula of $\gamma$. 


\section{Some final remarks}

We note that in the formulation of the Theorem 3 , we use a closed $\mathcal{N} \mathcal{I}$ derivation, rather than saying that there is a derivation of $\gamma$ from $\Gamma$ iff the sequent $\Gamma \vdash \gamma$ is derivable. The reason for this is the implicit treatment of the rule of contraction in $\mathcal{N} \mathcal{I}$. Due to that, only a contraction whose contraction formula is active, in a sense that it is a side formula of an operational rule in a sequent derivation, can be expressed in natural deduction. In an arbitrary derivation of $\Gamma \vdash \gamma$, we can guarantee that every contraction formula is active, only when $\Gamma$ is empty.

$\mathcal{N} \mathcal{I}$-derivations are normal, by the definition. As a consequence, they are not closed under composition (it is clear that a composition of normal derivations need not be a normal derivation). In spite of that, we can prove the following Closure Lemma, indirectly, via a proof of cut-elimination for the calculus GI.

Closure Lemma. If there is a derivation of $\alpha$ from $\Gamma$ in $\mathcal{N I}$ and there is a derivation of $\beta$ from $\alpha, \ldots, \alpha, \Delta$ in $\mathcal{N} \mathcal{I}$, then there is a derivation of $\beta$ from $\Gamma, \ldots, \Gamma, \Delta$ in $\mathcal{N} \mathcal{I}$.

Proof: Let $\mathcal{D}_{1}$ be a derivation of $\alpha$ from $\Gamma$ and let $\mathcal{D}_{2}$ be a derivation of $\beta$ from $\alpha, \ldots, \alpha, \Delta$ in $\mathcal{N} \mathcal{I}$. We use the translation given in the proof of the Theorem 3 , to translate $\mathcal{D}_{1}$ and $\mathcal{D}_{2}$ into normal GI-derivations $\begin{gathered}\pi_{1} \\ \Gamma \vdash \alpha\end{gathered}$ and $\frac{\pi_{2}}{\alpha, \ldots, \alpha, \Delta \vdash \beta}$. Every contraction in $\pi_{1}$ and $\pi_{2}$ is obtained by a translation of an operational rule with multiple discharge of assumptions. This means that every contraction formula in $\pi_{1}$ and $\pi_{2}$ is active.

Then, by eliminating multiple cut in:

$$
\begin{array}{cc}
\pi_{1} & \pi_{2} \\
\Gamma \vdash \alpha \quad \alpha, \ldots, \alpha, \Delta \vdash \beta & \text { (mcut) }
\end{array}
$$

we obtain a normal $G I$-derivation $\pi^{n}$ of $\Gamma, \ldots, \Gamma, \Delta \vdash \beta$. This derivation is also with active contraction formulae, only (the elimination procedure does not need to produce contractions with inactive formulae). This is crucial for our translation from a sequent calculus derivation of a sequent 
$\Gamma, \ldots, \Gamma, \Delta \vdash \beta$ to a natural deduction derivation of $\beta$ from the multiset of open assumptions $\Gamma, \ldots, \Gamma, \Delta$.

The translation from a sequent calculus derivation, of a sequent $\Gamma \vdash \gamma$, to a natural deduction derivation of $\gamma$, from the multiset of open assumptions $\Gamma$, is given inductively, as in the proof of the Theorem 3, with only difference that sequent-formulae without discharge label may appear here (they are denoted by $\Theta$ ). E.g.:

$\pi_{1}$

1.1. $\frac{\alpha, \Theta \vdash \beta}{\Theta \vdash \alpha \rightarrow \beta}(\rightarrow \mathrm{r}) \mapsto \frac{[\alpha]^{(1)}, \Theta \vdash \beta}{\alpha \rightarrow \beta}(\rightarrow \mathrm{I})^{(1)}$

$\pi_{1} \quad \pi_{2} \quad \pi_{1} \quad \pi_{2}$

1.2. $\frac{\Theta \vdash \alpha \quad \Theta \vdash \beta}{\Theta \vdash \alpha \wedge \beta}(\wedge \mathrm{r}) \mapsto \frac{\Theta \vdash \alpha \quad \Theta \vdash \beta}{\alpha \wedge \beta}(\wedge \mathrm{I})$

$\pi_{1} \quad \pi_{2}$

2.2. $\frac{\Gamma, \Theta_{1} \vdash \alpha \quad \Pi, \beta, \Theta_{2} \vdash \gamma}{[\Gamma],[\Pi],[\alpha \rightarrow \beta]^{(k)}, \Theta_{1}, \Theta_{2} \vdash \gamma}(\rightarrow 1) \mapsto$

$$
\begin{aligned}
& {[\alpha \rightarrow \beta]^{(k)} \quad \pi_{1} \quad \pi_{2}} \\
& \mapsto \frac{\overline{\alpha \rightarrow \beta} \quad[\Gamma], \Theta_{1} \vdash \alpha \quad[\Pi],[\beta]^{(i)}, \Theta_{2} \vdash \gamma}{\gamma}(\rightarrow \mathrm{E})^{(i)}
\end{aligned}
$$

$\pi_{1}$

$\pi_{2}$

2.6 .

$\frac{\Gamma, \alpha, \Theta \vdash \gamma \quad \Gamma, \beta, \Theta \vdash \gamma}{[\Gamma],[\alpha \vee \beta]^{(k)}, \Theta \vdash \gamma}(\vee 1) \mapsto$

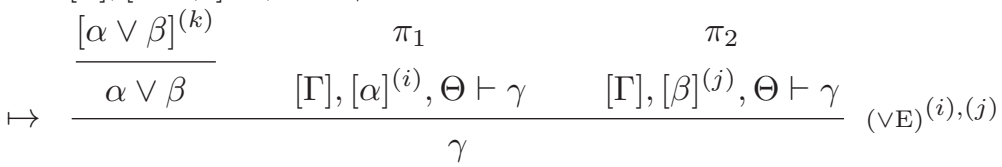

$\pi_{1}$

2.7. $\frac{\Gamma, \alpha, \alpha, \Theta \vdash \gamma}{[\Gamma],[\alpha]^{(k)}, \Theta \vdash \gamma}(\mathrm{WE}) \mapsto \quad[\Gamma],[\alpha]^{(k)},[\alpha]^{(k)}, \Theta \vdash \gamma$

3.1. $[\alpha]^{(k)},\left[\alpha_{1}\right]^{\left(k_{1}\right)}, \ldots,\left[\alpha_{n}\right]^{\left(k_{n}\right)}, \Theta \vdash \alpha \mapsto \frac{[\alpha]^{(k)},\left[\alpha_{1}\right]^{\left(k_{1}\right)}, \ldots,\left[\alpha_{n}\right]^{\left(k_{n}\right)}, \Theta}{\alpha}$ 
By this translation we obtain an $\mathcal{N} \mathcal{I}$-derivation of $\beta$ from $\Gamma, \ldots, \Gamma, \Delta$.

Finally, natural deduction calculi of some substructural logics can be obtained from $\mathcal{N} \mathcal{I}$, in a way we obtain their sequent calculus formulations from GI. E.g., by rejecting the rule (W) in $G I$, we obtain a contractionless logic whose natural deduction system can be obtained from $\mathcal{N} \mathcal{I}$ by forbidding multiple discharge of assumptions. Or, by restricting the axioms in $G I$ to:

$$
\alpha \vdash \alpha \quad \text { and } \quad \perp \vdash \gamma
$$

we obtain a weakening-less logic, whose natural deduction system can be obtained from $\mathcal{N} \mathcal{I}$ by restricting initial rules to rules of the following forms, only:

$$
\frac{\alpha}{\alpha} \quad \text { and } \quad \frac{\perp}{\gamma} \text {. }
$$

The former is the $B C K$ logic and the later one is the distribution-less relevant logic. The problems of similar nature are discussed also in [7], however, on the basis of the calculi which are different from ours.

\section{Acknowledgments}

I would like to thank the referees for very helpful comments and useful suggestions.

\section{References}

[1] K. Došen, A Historical Introduction to Substructural Logics, Substructural Logics, (eds. P. Schroeder-Heister and K. Došen), pp. 1-30, Oxford Science Publication, (1993).

[2] N. Francez, Relevant harmony, Journal of Logic and Computation, Volume 26, Number 1 (2016), pp. 235-245.

[3] G. Gentzen, Investigations into logical deduction, The Collected Papers of Gerhard Gentzen, Szabo, M. E. (ed.) North-Holland, pp. 68-131, (1969).

[4] S. Negri, A normalizing system of natural deduction for intuitionistic linear logic, Archive for Mathematical Logic 41 (2002), pp. 789-810. 
[5] S. Negri, J. von Plato, Sequent calculus in natural deduction style, The Journal of Symbolic Logic, Volume 66, Number 4 (20011), pp. 1803-1816.

[6] J. von Plato, Natural deduction with general elimination rules, Archive for Mathematical Logic 40 (2001), pp. 541-567.

[7] G. Restall, Proof theory \& philosophy, manuscript, available at http://consequently.org/writing/ptp

[8] M. H. Sørensen, P. Urzyczyn, Lectures on the Curry-Howard Isomorphism, Studies in Logic and the Foundations of Mathematics, Volume 149 (2006), Elsevier.

[9] A. S. Troelstra, H. Schwichtenberg, Basic Proof Theory, Cambridge University Press, (1996).

University of Belgrade

Faculty of Economics

Kamenička 6

11000 Belgrade, Serbia

e-mail: mirjanailic@ekof.bg.ac.rs 